ISSN 2179-6750

\title{
Avaliação da concordância entre informações obtidas com questionário aplicado a usuários de uma unidade de saúde de Porto Alegre e as disponíveis nos prontuários
}

Evaluation of the agreement between information obtained from questionnaire applied to users of a Porto Alegre's health unit and available in the medical records

Evaluación del acuerdo entre la información obtenida a partir del cuestionario aplicado a los usuarios de una unidad de salud en Porto Alegre y las disponibles en los registros médicos

\section{Sílvia Pauli ${ }^{1}$; Lena Azeredo de Lima ${ }^{2}$; Eno Dias de Castro Filho ${ }^{3}$}

\begin{abstract}
Resumo
Analisar a concordância entre as informações obtidas a partir das respostas do questionário de uma pesquisa com informações disponíveis nos prontuários pode auxiliar na definição da confiabilidade dos resultados em que se baseiam suas conclusões. Técnicas estatísticas consideradas adequadas para verificar concordâncias são as fórmulas KAPPA e PABAK. Verificou-se a concordância das informações obtidas através do questionário de linha de base com as obtidas pela pesquisa nos prontuários dos pacientes. Tratou-se de um estudo de concordância. Foram analisadas 15 perguntas, das 93. As informações obtidas através das duas fontes foram comparadas para estimar a magnitude de concordância. Foi calculada a estatística kappa ou PABAK através do software Winpepi versão 11.25. Foram analisados questionários de 149 indivíduos com média de idade de 62,64 \pm 13,35 anos, sendo $69,12 \%$ do sexo feminino. As concordâncias para a maioria das perguntas foram classificadas como substanciais. Os resultados sugerem que as informações obtidas com o questionário do estudo "Avaliação da Atenção à Saúde em Hipertensão e Diabete em Atenção Primária" estejam refletindo a realidade estudada.
\end{abstract}

Descritores: Avaliação, Concordância entre informações, Questionário, Prontuário médico.

\begin{abstract}
To analyze the correlation between the information obtained from the responses to the questionnaire of research, with information available in the medical records, can help to define the reliability of the results that support their conclusions. Statistical techniques considered adequate to verify concordances are kappa and PABAK formula. To verify the concordance between the information obtained from the baseline questionnaire with those obtained by searching through the patients records. It was a concordance study. Fifteen questions were analyzed, out of 93. The information obtained from the two sources was compared to estimate the magnitude of agreement. We calculated the kappa or PABAK statistic through the Winpepi software version 11.25. Questionnaires from 149 individuals, with middle age of $62.64 \pm 13.35$ years, $69.12 \%$ from which female were analyzed. The concordance of most questions was classified as substantial. The results suggest that the information obtained with the study "Evaluation of Health Care for Hypertension and Diabetes in Primary Care" questionnaire are reflecting the studied reality.
\end{abstract}

\footnotetext{
${ }^{1}$ Grupo Hospitalar Conceição. Porto Alegre, RS, Brasil. email: silviaspauli@ gmail.com

${ }^{2}$ Grupo Hospitalar Conceição. Porto Alegre, RS, Brasil. email: lenut68@ gmail.com

${ }^{3}$ Grupo Hospitalar Conceição. Porto Alegre, RS, Brasil. email: enofilhouol@ gmail.com
} 
ISSN 2179-6750

Key-words: Evaluation, Correlation between Information, Questionnaire, Medical Records.

\section{Resumen}

Analizar la correlación entre la información obtenida de las respuestas al cuestionario de una encuesta de la información disponible en los registros médicos pueden ayudar en la definición de la confiabilidad de los resultados que soportan sus conclusiones. Las técnicas estadísticas que considere convenientes para comprobar las concordancias son las fórmulas kappa y PABAK. Fue hecho el acuerdo de la información obtenida mediante el cuestionario de referencia con los obtenidos por la investigación en los registros médicos de los pacientes. Este fue un estudio de concordancia. Se analizaron 15 preguntas, de las 93. La información obtenida de las dos fuentes se compararon para estimar la magnitud del acuerdo. Se calculó el índice kappa o PABAK a través de software winpepi versión 25.11. Los cuestionarios fueron analizados en 149 pacientes con una edad media de 62,64 \pm 13,35 años y 69,12\% mujeres. Los acuerdos para la mayoría de las preguntas se clasificaron como sustancial. Los resultados sugieren que la información obtenida de la "Evaluación de la Salud Atención de hipertensión y diabetes en atención primaria" cuestionario de estudio están reflejando la realidad estudiada.

Palabras-claves: Evaluación, Concordancia entre la Información, Cuestionario, Registros Médicos.

\section{Introdução}

O conhecimento científico se desenvolve, em grande medida, a partir de mensurações (medidas). Por trás da medida pode haver erros devido ao observador, ao instrumento (ou método) ou ao momento da observação. Daí advem potenciais vieses ou um aumento na imprecisão do fenômeno estudado. Portanto, faz-se necessário conhecer quão confiável é a mensuração realizada ${ }^{1}$.

Pode-se definir confiabilidade de um experimento, teste ou medição como a capacidade de obter resultados com variações mínimas, quando realizada por diferentes pessoas ou em diferentes momentos ${ }^{2}$.

Em 2011 teve início o estudo longitudinal denominado: “Avaliação da Atenção à Saúde em Hipertensão e Diabete em Atenção Primária" do Centro de Estudo e Pesquisa em Atenção Primária do Grupo Hospitalar Conceição (GHC), que tem o objetivo de melhorar o atendimento e a meta de identificar, monitorar e avaliar as ações de saúde de maior impacto para promover saúde, prevenir, tratar e recuperar as complicações de Hipertensão Arterial Sistêmica (HAS) e Diabetes Mellitus (DM) na Atenção Primária. É um estudo longitudinal com previsão de, no mínimo, 4 anos de duração e tem como cenário o Serviço de Saúde Comunitária (SSC) do GHC, no município de Porto Alegre, abrangendo as doze unidades de saúde, que, ao todo, são referência para cerca de 108 mil habitantes. A Unidade de Saúde Barão de Bagé (USBB), referência para cerca de 4 mil habitantes, é uma das doze unidades mencionadas.

Foram entrevistados 2.482 moradores da área de abrangência das 12 Unidades de Saúde do SSC, com 18 anos de idade ou mais, usuários do SSC com diagnóstico de HAS e/ou DM, que 
ISSN 2179-6750

tenham efetuado pelo menos uma consulta nos três anos anteriores à pesquisa com registro de HAS ou DM (68\% mulheres; 50\% com menos de 65 anos de idade).

Checar seus resultados com outra fonte considerada adequada para ao menos uma série de questões (prontuário) pode contribuir para determinar o grau de confiança nos mesmos. Haverá maior confiança em destinar os recursos necessários à implementação das recomendações de uma pesquisa (com vistas aos desfechos buscados) se houver alta confiança na capacidade de seus instrumentos em detectar e medir a realidade estudada.

Uma técnica estatística considerada adequada para verificar concordâncias entre informações obtidas de fontes diferentes é a fórmula kappa, mas em algumas situações pode-se recorrer a outras técnicas.

Segundo Petrie e Sabin ${ }^{3}$, o kappa é dependente tanto do número de categorias quanto da prevalência da situação. Por isso, é necessário que se tenha atenção na comparação de kappas de estudos diferentes.

Alguns autores, como Tanner e Young ${ }^{4}$ já escreveram sobre a dependência do kappa em relação à prevalência da característica em estudo, ou seja, kappa é afetado de forma complexa pelas distribuições de dados entre as categorias que são usadas ("prevalência") ${ }^{5}$. Assim, valores paradoxais de kappa (inconsistência com a concordância aparente) podem ocorrer. $\mathrm{O}$ cálculo do Kappa utiliza a proporção de azar, ou concordância esperada, que pode ser interpretada como a proporção de vezes em que os avaliadores concordam por acaso. Ainda que haja grande concordância geral observada, o coeficiente kappa não é bom com prevalências muito baixas ou muito altas da condição avaliada, pois nesses casos a concordância esperada pelo acaso será muito grande ${ }^{6}$. Levando em consideração a tendência de a prevalência influenciar na determinação da magnitude do coeficiente kappa (viés de prevalência), um valor ajustado, o PABAK (Prevalence and Bias Ajusted Kappa), é utilizado ${ }^{7}$. PABAK é o valor que kappa teria se não houvesse influência da prevalência de dados entre as categorias.

O objetivo do presente estudo é avaliar o grau de concordância das informações obtidas no questionário de linha de base do estudo "Avaliação da Atenção à Saúde em Hipertensão e Diabete em Atenção Primária" com as informações que se encontram nos prontuários dos pacientes da Unidade de Saúde Barão de Bagé.

\section{Métodos}

Este é um estudo de concordância entre informações provenientes de duas fontes: o subconjunto referente à Unidade de Saúde Barão de Bagé do banco de dados da linha de base do 
ISSN 2179-6750

estudo "Avaliação da Atenção à Saúde em Hipertensão e Diabetes em Atenção Primária" e os prontuários dos mesmos pacientes na referida Unidade de Saúde.

A população e amostra do estudo são moradores da área de abrangência da Unidade de Saúde Barão de Bagé do SSC, que foram entrevistados no estudo "Avaliação da Atenção à Saúde em Hipertensão e Diabetes em Atenção Primária”, totalizando 156 indivíduos.

Do banco de dados, foram selecionadas perguntas cujas informações foram julgadas possíveis de serem encontradas também nos prontuários das pessoas. De um total de 93 perguntas, $15(16 \%)$ foram incluídas para análise, as quais constam na tabela 2, na sessão "Resultados".

A partir do número de registro do GHC (número destinado aos pacientes que ingressam nas unidades do GHC, no ato do cadastro), também constante no banco de dados, buscou-se o prontuário de cada indivíduo.

Foi analisado se há concordância entre a informação obtida no questionário e a informação constante no prontuário, registrada por profissionais de saúde. Como os dados das duas fontes não estão expressos do mesmo modo, criou-se para cada pergunta uma instrução de leitura e/ou transformação voltada a produzir uma informação comparável.

Para análise de algumas informações, seus resultados foram dicotomizados de modo a que expressassem a intenção de busca de conhecimentos pelos pesquisadores da linha de base, obtida junto aos mesmos pesquisadores mediante entrevista aberta. Isso ocorreu para as perguntas: “Quantas vezes o(a) Sr(a) consultou com o médico, por pressão alta, na sua Unidade de Saúde de Referência?" e "Quantas vezes o(a) Sr(a) consultou com o médico, por diabete ou açúcar alto no sangue, na sua Unidade de Saúde de Referência?” As respostas foram dicotomizadas para expressar se os entrevistados haviam realizado ao menos uma consulta médica nos últimos 6 meses ou não. As perguntas "Há quantos meses foi sua última consulta médica, devido à diabete ou açúcar alto no sangue, na sua Unidade de Saúde de Referência?" e "Há quantos meses foi sua última consulta médica, devido à pressão alta, na sua Unidade de Saúde de Referência?" tinham o objetivo de aferir a consistência das respostas às anteriores e suas respostas também foram dicotomizadas para expressar se houve ou não ao menos uma consulta neste período, com tolerância de diferenças de até 30 dias, para fins de não detectar falta de concordância artificial devido a vieses de memória do entrevistado. Ou seja, se o usuário respondeu que essa consulta foi há dois meses (sessenta dias), considerou-se como concordância o prazo entre 30 e 90 dias da data da última consulta, conforme registro no prontuário.

Para a pergunta "Há quantos meses foi sua última consulta com o dentista na sua Unidade de Saúde de Referência", foi adotado o mesmo prazo de tolerância, pelos mesmos motivos. 
ISSN 2179-6750

A consulta às informações nos prontuários dos pacientes foi realizada sobre o período a que se referiam as respostas aos questionários da linha de base, que foram obtidas entre março e abril de 2011.

Para extração dos dados, foi elaborada uma planilha eletrônica para registro das seguintes variáveis: número do registro no GHC; número do prontuário na USBB; número do questionário; data da entrevista; idade; sexo. Essa planilha também contou com uma coluna para cada pergunta, com subcolunas onde foram registradas as informações das duas fontes dos dados, observações úteis para melhorar a compreensão sobre qualquer uma das fontes e então permitir um melhor julgamento sobre concordância e uma atribuição de sim ou não para concordância.

As informações obtidas através do questionário do estudo "Avaliação da Atenção à Saúde em Hipertensão e Diabetes em Atenção Primária" e as informações obtidas nos prontuários dos entrevistados foram comparadas de modo a se estimar a magnitude da concordância. A definição "sim” para acordo corresponde à informação igual nas duas fontes. Foi calculada a estatística kappa, com intervalo de confiança de 95\%, ou PABAK, para a qual não é possível gerar intervalo de confiança, através do software Winpepi versão 11.25. Para 7 perguntas com prevalência elevada de uma resposta, foi calculado o PABAK, a fim de corrigir os valores paradoxais causados pela prevalência. Para as demais 8 perguntas, calculou-se índice kappa. Os pontos de corte utilizados foram os sugeridos por Petrie e Sabin ${ }^{3}$ : Concordância pobre se k até 0,20; justa (leve) se k entre 0,21 e 0,40 ; moderada se k entre 0,41 e 0,60 ; substancial se $\mathrm{k}$ entre 0,61 e 0,80 ; boa (excelente) se $\mathrm{k}$ $>0,80$.

Este estudo foi aprovado pelo Comitê de Ética em Pesquisa do Grupo Hospitalar Conceição (CEP-GHC).

\section{Resultados}

De 156 questionários do banco de dados de linha de base foram excluídos sete indivíduos cujos prontuários não foram localizados na USBB. O número de sujeitos com informações nas duas fontes (questionário do estudo e prontuário) foi de 149, com média de idade de 62,64 \pm 13,35 anos, sendo $69,12 \%(\mathrm{n}=103)$ do sexo feminino.

$\mathrm{Na}$ tabela 1 é possível verificar que 6 pessoas pensavam ter somente uma das morbidades (HAS ou DM), quando, de acordo com os registros no prontuário, possuíam as duas, HAS e DM. 
Tabela 1. Número de sujeitos com Hipertensão e/ou Diabete, conforme as duas fontes.

\begin{tabular}{cccc}
\hline & HAS* $(\mathbf{n})$ & DM** (n) & HAS+DM (n) \\
\hline Questionário & 95 & 10 & 44 \\
Prontuário & 91 & 8 & 50 \\
TOTAL & \multicolumn{4}{c}{149} & \\
\hline
\end{tabular}

A tabela 2 apresenta a análise de concordância entre as fontes, com as estatísticas. Foram elevadas as concordâncias para as 15 perguntas estudadas. Onze perguntas $(73,33 \%)$ tiveram a concordância classificada como substancial, ou seja kappa ou PABAK entre 0,61 e 0,80. Para 4 perguntas $(26,66 \%)$ a concordância foi classificada como excelente, ou seja, kappa ou PABAK $>0,80$. Os maiores índices de concordância foram encontrados para as perguntas " $\mathrm{O}(\mathrm{A}) \operatorname{Sr}(\mathrm{a})$. precisa usar remédio(s) para pressão alta?” $(\mathrm{k}=0,98)$ e “ $\mathrm{O}(\mathrm{A}) \operatorname{Sr}(\mathrm{a})$. usa remédio para pressão alta, ou algum médico já lhe disse que o(a) Sr(a). tem problema de pressão alta?” $(\mathrm{k}=0,97)$. As concordâncias mais baixas foram observadas para as perguntas "quantas vezes você consultou com o médico devido à diabetes ou açúcar alto no sangue na sua Unidade de Saúde de Referência?" $(\mathrm{k}=0,64)$ e "Há quantos meses foi

sua última consulta com o dentista na sua Unidade de Saúde de Referência?” $(\mathrm{k}=0,62)$. No entanto, mesmo sendo as de menor concordância, ainda assim estão classificadas como tendo concordância substancial.

No que diz respeito ao preenchimento de informações nos prontuários, para a pergunta "Você fuma ou já fumou?", foi verificada ausência de informação a este respeito em 7,38\% (n=11) do conjunto de prontuários analisados. Isso foi interpretado como não concordância, mas não representa necessariamente uma debilidade do banco de dados de linha de base, mas provavelmente dos registros nos prontuários. Outra dificuldade encontrada ao longo da análise dos prontuários foi a falta de legibilidade das evoluções de alguns profissionais de saúde. 


\section{ISSN 2179-6750}

Tabela 2. Concordância entre informações dos questionários aplicados aos usuários da USBB* e dos prontuários dos mesmos.

\begin{tabular}{|c|c|c|c|c|}
\hline PERGUNTA & $\begin{array}{c}\text { \% DE } \\
\text { CONCORDÂNCIA }\end{array}$ & KAPPA & IC** 95\% & PABAK $* * *$ \\
\hline Qual é sua data de nascimento? & $94,63 \%(n=141)$ & 0,89 & $0,82-0,96$ & \\
\hline $\begin{array}{l}\text { O (A) Sr(a). usa remédio para pressão alta, } \\
\text { ou algum médico já lhe disse que o(a) } \\
\text { Sr(a). tem problema de pressão alta? }\end{array}$ & $98,65 \%(n=147)$ & & & 0,97 \\
\hline $\begin{array}{l}\text { O (A) } \operatorname{Sr}(\text { a). usa remédio para diabete ou } \\
\text { açúcar alto no sangue, ou algum médico já } \\
\text { lhe disse que o(a) } \operatorname{Sr}(\text { a). tem problema de } \\
\text { diabete ou açúcar alto no sangue? }\end{array}$ & $95,97 \%(n=143)$ & 0,91 & $0,85-0,98$ & \\
\hline $\mathrm{O}(\mathrm{A}) \operatorname{Sr}(\mathrm{a})$. fuma ou já fumou? & $85,90 \%(n=128)$ & 0,78 & $0,70-0,86$ & \\
\hline Mais alguém na sua casa tem pressão alta? & $86,57 \%(n=129)$ & 0,73 & $0,62-0,84$ & \\
\hline $\begin{array}{l}\mathrm{O}(\mathrm{A}) \mathrm{Sr}(\mathrm{a}) \text {. precisa usar remédio(s) para } \\
\text { pressão alta? }\end{array}$ & $97,98 \%(n=146)$ & & & 0,98 \\
\hline $\begin{array}{l}\text { Desde (seis meses atrás) quantas vezes } \\
\text { o(a) Sr(a). consultou com o médico por } \\
\text { pressão alta na sua Unidade de Saúde de } \\
\text { Referência? }\end{array}$ & $79,86 \%(n=119)$ & & & 0,72 \\
\hline $\begin{array}{l}\text { Desde (seis meses atrás) há quantos meses } \\
\text { foi sua última consulta médica devido à } \\
\text { pressão alta na sua Unidade de Saúde de } \\
\text { Referência? }\end{array}$ & $81,20 \%(\mathrm{n}=121)$ & & & 0,78 \\
\hline $\begin{array}{l}\text { Essa consulta foi agendada ou marcada } \\
\text { com antecedência? }\end{array}$ & $75,16 \%(n=112)$ & 0,68 & $0,54-0,83$ & \\
\hline $\begin{array}{l}\text { Mais alguém na sua casa tem diabete ou } \\
\text { açúcar alto no sangue? }\end{array}$ & $93,95 \%(n=140)$ & 0,73 & $0,60-0,87$ & \\
\hline $\begin{array}{l}\text { Desde (seis meses atrás) quantas vezes } \\
\text { o(a) Sr(a). consultou com o médico por } \\
\text { diabete ou açúcar alto no sangue na sua } \\
\text { Unidade de Saúde de Referência? }\end{array}$ & $90,60 \%(n=135)$ & & & 0,64 \\
\hline $\begin{array}{l}\text { Desde (seis meses atrás), há quantos } \\
\text { meses foi sua última consulta médica } \\
\text { devido a diabete ou açúcar alto no sangue } \\
\text { na sua Unidade de Saúde de Referência? }\end{array}$ & $91,94 \%(n=137)$ & & & 0,75 \\
\hline $\begin{array}{l}\text { Essa consulta foi agendada com } \\
\text { antecedência? }\end{array}$ & $89,93 \%(n=134)$ & & & 0,79 \\
\hline $\begin{array}{l}\text { Desde (seis meses atrás), quantas vezes } \\
\text { o(a) Sr(a). consultou com o dentista, por } \\
\text { qualquer motivo, na sua Unidade de Saúde } \\
\text { de Referência? }\end{array}$ & $77,18 \%(\mathrm{n}=115)$ & 0,66 & $0,55-0,76$ & \\
\hline $\begin{array}{l}\text { Desde (seis meses atrás), há quantos } \\
\text { meses foi a sua última consulta com o } \\
\text { dentista na sua Unidade de Saúde de } \\
\text { Referência? }\end{array}$ & $80,53 \%(n=120)$ & 0,62 & $0,49-0,74$ & \\
\hline
\end{tabular}

*USBB = Unidade de Saúde Barão de Bagé; **IC = Intervalo de Confiança; ***PABAK = Prevalence and Bias Ajusted Kappa 


\section{Discussão}

A análise da concordância entre as informações nos questionários do estudo "Avaliação da Atenção à Saúde em Hipertensão e Diabetes em Atenção Primária" com aquelas disponíveis nos prontuários demonstrou concordância substancial e excelente para as perguntas estudadas. Tais resultados indicam que os dados do questionário estejam refletindo a realidade estudada, podendo ser consideradas confiáveis. A avaliação dos instrumentos utilizados em pesquisa permite qualificar e confirmar os dados obtidos, possibilitando, assim, que os resultados possam refletir melhor a realidade.

No presente estudo, observaram-se problemas relacionados ao preenchimento dos prontuários, como ausência de informação sobre tabagismo, além de falta de legibilidade das evoluções de alguns profissionais de saúde. Müller ${ }^{8}$ afirma que o desenvolvimento de um prontuário ideal deve abranger e conter todos os dados relevantes em relação a informações pessoais, história de saúde geral, passada e atual, além de anotações referentes a observações realizadas pelo profissional de saúde e exames físicos efetuados. Galvão et al ${ }^{9}$ referem alguns problemas de registros em prontuários encontrados ao longo de sua pesquisa, entre eles informação incompleta e até mesmo omissão do nome do profissional da saúde responsável pelo atendimento, além de ausência da data de atendimento.

Campos et al ${ }^{10}$ conduziram um estudo com o objetivo de avaliar a qualidade das informações do projeto de investigação "Estudo da morbi-mortalidade e da atenção Peri e Neonatal no Município do Rio de Janeiro", analisando também a concordância das informações coletadas de diferentes fontes (entrevista com as puérperas e prontuários médicos), utilizando a estatística kappa e puderam demonstrar que as informações colhidas das duas fontes tiveram substancial concordância, sugerindo alta estabilidade das informações coleadas no referido projeto.

Theme Filha et al ${ }^{11}$, a partir de uma pesquisa realizada referente à confiabilidade do Sistema de informações sobre nascidos vivos hospitalares no município do Rio de Janeiro, encontraram concordância excelente para as variáveis maternas analisadas. $\mathrm{O}$ estudo assinalou o enorme potencial da utilização de bases de dados que podem ser trabalhadas conjuntamente utilizando o cruzamento de informações provenientes de várias origens tais como inquéritos de saúde, informações hospitalares, sistemas de informações sobre mortalidade. Por outro lado ressaltam a importância do investimento intensivo na qualificação de suas informações.

Já no estudo de Sarinho et al ${ }^{12}$, que estimou a fidedignidade da Declaração de Óbito (DO) em relação a cada causa básica de óbito em quatro maternidades públicas da cidade do Recife, PE, e avaliou a correlação entre dados do prontuário e da DO através do índice de Kappa, foi 15 
ISSN 2179-6750

demonstrada concordância regular, sofrível ou fraca para a maioria das categorias estudadas. Isso evidencia que nem sempre os questionários são desenhados de modo a obter informações confiáveis ou que os prontuários podem conter registros de muito baixa qualidade.

Ao compararem-se informações do estudo "Avaliação da Atenção à Saúde em Hipertensão e Diabetes em Atenção Primária" com as de outra fonte considerada adequada para ao menos uma série de questões (prontuário), e demonstrar que estas apresentam, em geral, substancial concordância, indicando que estejam refletindo a realidade estudada, conclui-se que há alta estabilidade das informações coletadas a partir do questionário do referido estudo, gerando confiança na informação dali obtida.

\section{Referências}

1. Luiz RR. Métodos Estatísticos em Estudos de Concordância. In: Medronho RA, Bloch KV, Luiz RR, Werneck GL. Epidemiologia. 2. Ed. São Paulo: Atheneu, 2009. 685 p.

2. Almeida Filho N. Epidemiologia sem números: uma introdução crítica à ciência epidemiológica. Ed. Campus, Rio de Janeiro, 1989. 105p.

3. Petrie A, Sabin C. Compêndio de estatística médica. Lisboa Inst. Piaget, c2000. 342 p.

4. Tanner MA, Young MA. Modelling agreement among raters. J. Am. Stat. Assoc., 80:175$80,1985$.

5. Byrt T, Bishop J, Carlin JB. Bias, Prevalence and Kappa. Journal of Clinical Epidemiology, volume 46, Issue 5, May 1993, pages 423-429.

6. Gjorup T. The kappa coefficient and the prevalence of a diagnosis. Methods Inf Med. 1988, 27(4):184-6.

7. Sim J, Wright CC. The Kappa Statistic in Reability Studies: Use, Interpretation and Sample Size Requirements. Phy Ther. 2005; 85:257-268. Downloaded from http://ptjournal.apta.org/ by guest on November 11, 2013.

8. Müller MP. A busca do prontuário ideal. Disponível em http://www.portaleducacao.com.br/odontologia/artigos/2875/a-busca-do-prontuario-ideal. Acesso em: 28 de set. de 2013.

9. Galvão MCB, Silva JC, Ferreira VS, Rocha JES. Linguagens empregadas em prontuários do paciente frente aos processos de organização e recuperação da informação no contexto da saúde. Disponível em http://www.ancib.org.br/media/dissertacao/3516.pdf. Acesso em: 28 de set. de 2013. 
ISSN 2179-6750

10. Campos MR, Leal MC, Souza JPR, Cunha CB. Consistência entre fontes de dados e confiabilidade interobservador do Estudo de Morbi-mortalidade e Atenção Peri e Neonatal no Município do Rio de Janeiro. Cad. Saúde Pública, Rio de Janeiro, 20 Sup 1:S34-S43, 2004.

11. Theme MM, Gama SGN, Cunha CB, Leal MC. Confiabilidade do Sistema de Informações sobre nascidos vivos hospitalares no município do Rio de Janeiro, 1999-2001. Cad. Saúde Pública, Rio de Janeiro, 20 Sup 1:S83-S91, 2004.

12. Sarinho SW, Coutinho SB, Acioli TML, Menezes TF, Abreu MAP, Lacerda N, Guimarães MJB. Mortalidade neonatal em Recife, PE: causas básicas e grau do conhecimento dos neonatologistas acerca do preenchimento das declarações de óbito. Pediatria (São Paulo), 23(4):279-84; 2001. 